\title{
ON THE CAPACITY OF CELLULAR FIXED RELAY NETWORKS
}

\author{
Hakan Bolukbasi ${ }^{*}$, Halim Yanikomeroglu*, David D. Falconer ${ }^{*}$, Shalini Periyalwar ${ }^{* *}$ \\ ${ }^{*}$ Broadband Communication \& Wireless Systems (BCWS) Centre \\ Dept. of Systems \& Computer Engineering \\ Carleton University, Ottawa, Canada \\ ${ }^{* *}$ Nortel Networks, Ottawa, Canada
}

\begin{abstract}
Augmentation of the conventional cellular networks with wireless fixed relays, to enable multi-hop communications, is being considered for beyond-3G cellular networks in order to provide high data rate coverage in a cost-effective manner. In this paper, we consider a Cellular Relay Network (CRN) where a large number of low-complexity/low-power wireless fixed relays are deployed around the central node (equivalent of a BS) in each cell. Each relay and central node covers only a small area with a small amount of power, enabling the reuse of the frequency-time resources. The contribution of this paper is to evaluate the cell capacity (i.e., aggregate throughput) in the CRN which enables to make comparisons between the cell capacities of the CRN and the conventional cellular network. The paper shows that the cell capacity of the CRN does not depend on the cell size or the total number of relays in the cell, but it rather depends on the number of nearest relays to the central node.
\end{abstract}

\section{INTRODUCTION}

Future wireless systems are expected to provide very high data rates; it is estimated that, for beyond $3 \mathrm{G}$ systems, peak data rate demands for mobile access and nomadic/local area wireless access could be around $100 \mathrm{Mbps}$ and $1 \mathrm{Gbps}$, respectively [1]. With the existing cellular architecture, this goal cannot be realized over a wide coverage area. Therefore, some novel cellular network architectures are being studied.

Pico-cell networks idea is one of the remedies to high data rate demand. Since the resources, i.e. frequency and time, for wireless communication are very scarce, by splitting macro-cells into much smaller sized cells, namely pico-cells, spectrum efficiency can be improved significantly [2]. Although the cost of base stations in pico-cellular network may be much less than that of conventional base stations, the very fact that each picocell base station would still require high-speed connection to the Internet makes this architecture not as cost effective as would be desired.

The multi-hop relaying concept may be considered as an alternate solution [3]. Relays are much simpler devices than base stations and they are not connected to the wired network; therefore, they are very cost effective. Adding relays in the cells reduces the signal transmission distances, resulting in lower propagation loss and higher average SNR to the mobile user.

Cellular Relay Networks is generalized form of the relaying concept. The whole cell area is covered by large number of relays. Each relay serves a small area with a small amount of power. Due to the low power feature, when there is sufficient electromagnetic separation, it is possible to reuse the channels (time-frequency resources) even in the same cell. Therefore, system performance can be improved from both reduced propagation loss and reusing the available channels.

\section{ARCHITECTURE}

Figure 1 shows a possible tree structure for CRN. The solid lines in the figure are considered as wireless links. Each dot represents a node or equivalently a relay; in this paper, those terms are used interchangeably. As seen from the figure, CRN may be considered as a combination of a central node (BS) and several trees, whose nodes are fixed relays; for simplicity, it is assumed that all trees are binary trees, i.e. each node has one parent node and two child nodes. The roots of all trees are positioned on a ring around the central node.

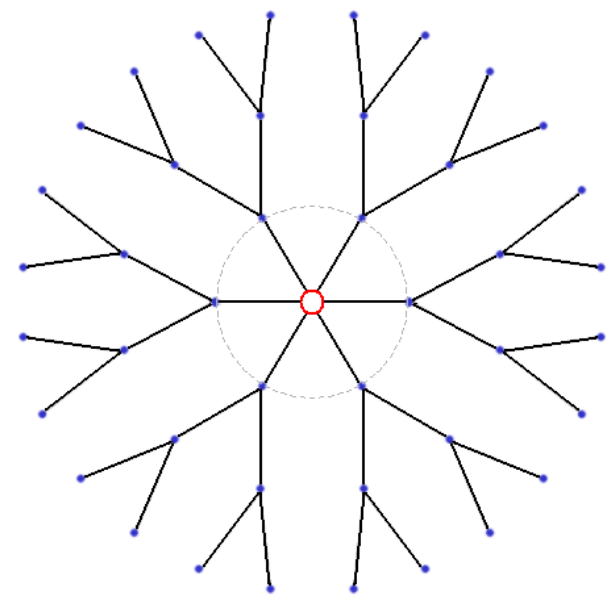

Figure 1. CRN configuration

All of the nodes are placed at positions where they have good links with their parent and child nodes. Nodes have very directional narrow beam antennas (such as pencil-beam antennas), for point-to-point communications. In other words, each node has three beam antennas; two for its child nodes and one for its parent node, with the exception of the leaf node (relays on the cell boundary) having only one for its parent node. In addition to the directional antennas, each node has an omnidirectional antenna to communicate with the users in its coverage area. 
The central node, unlike the relay nodes, has a connection to the wired network. It has as many directional antennas as the number of the root nodes. This reduces the interference among primary links emanating from the central node. This feature enables to use same bandwidth in all those links. Similar to relays, the central node uses an omni-directional antenna to communicate with the users in its coverage area.

Each node is responsible for the traffic in its coverage area and the traffic that needs to be relayed through it, i.e. traffic for the sub-tree originating from itself. For the downlink, each node gets traffic of its own and traffic that has to be relayed to its child nodes from its parent node. For the uplink, each node gets traffic from its child nodes and relays it to its parent node along with its own traffic.

Since the cell area is covered by a large number of relays, there is no need for a high power central node. Actually, if the number of relays is high enough, it may be assumed that all relays and the central node have approximately the same coverage area. Therefore, transmit power of the central node can be reduced to that of a relay. Since coverage areas of central node and the relays are considered to be equal, available bandwidth for central node and other nodes can be taken as equal. Although features of a central node and a relay are almost same, there is one fundamental difference: the central node has a connection to the wired network, whereas relays have no wired access to internet.

\section{CAPACITY OF THE CRN}

In this paper the term "capacity" refers to the aggregate throughput.

The central node has a direct link with all of the root nodes. Since all of those links use separate directional antennas, the same bandwidth can be used for all of them. Therefore, if the capacity of one link is found, others would also be the same. Figure 2 shows the central node and nodes belonging to one of the trees.

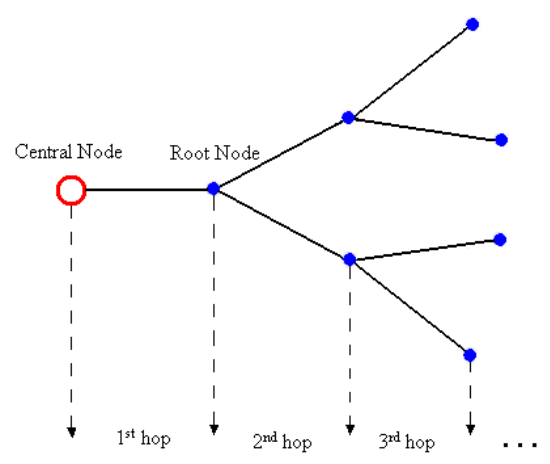

Figure 2. Central node and a tree

The traffic of all nodes (relays) that belong to the same tree must pass through the link between the central node and the root node, namely the primary link of that tree. Therefore, the capacity of a tree is limited by the capacity of the corresponding primary link.
Let $W$ be the total available bandwidth per cell in $\mathrm{Hz}$, and let $R$ be the capacity per cell in bits/sec. We will denote the cell capacity of the conventional cellular network, $\mathrm{CCN}$ (which does not employ any relays), as $R_{C C N}=R_{W} \mathrm{bits} / \mathrm{sec}$, where $R_{W}$ indicates that the capacity is a function of $W$.

Next, consider a CRN cell with $M$ root nodes each of which has $N$ nodes connected to it in a binary tree structure (Fig. 2). We assume that $B \mathrm{~Hz}$ of bandwidth is required to support the capacity of one relay, which is denoted by $R_{B}$. If the capacity of the central node (BS) is also equal to that of a relay, then

$$
R_{C R N}=(M \cdot N+1) R_{B} \approx M \cdot N \cdot R_{B}
$$

In order to compare $R_{C R N}$ with $R_{C C N}, R_{B}$ has to be found in terms of $R_{W}$, or equivalently $B$ has to be found in terms of $W$. As previously stated, total bandwidth, $W$, is fully used in each tree and is not equal to the bandwidth assigned to a primary link, since some portion of the total bandwidth is assigned for relayto-relay communication.

Assume that the total available bandwidth $W$ is divided into channels of $B \mathrm{~Hz}$. As stated above, each relay uses an average bandwidth of $B \mathrm{~Hz}$ in its coverage area.

Let us consider one tree. We define the following sets in a k-hop network:

$C:\{$ set of all channels available in a cell $\}$

$\boldsymbol{C}_{\boldsymbol{0}}:\{$ set of channels used by nodes in their coverage area\}

$C_{i}:\left\{\right.$ set of channels used by $\mathrm{i}^{\text {th }}$ hop links $\}, i=1,2, \ldots, k$

Note that,

$$
\begin{aligned}
& \boldsymbol{C}=\boldsymbol{C}_{0} \cup \boldsymbol{C}_{1} \cup \boldsymbol{C}_{2} \cup \ldots \cup \boldsymbol{C}_{\boldsymbol{k}} \\
& s(\boldsymbol{C})=W / B
\end{aligned}
$$

In the above, $s(\boldsymbol{C})$ stands for the size (cardinality) of the set $\boldsymbol{C}$; also, without loss of generality, we assumed that $W / B$ is an integer. To simplify the calculations, assume that all nodes (including central node) use the same channel in their coverage area. Then,

$$
s\left(\boldsymbol{C}_{0}\right)=1 \text { channel. }
$$

In order not to generate excessive interference, the channel in set $\boldsymbol{C}_{\boldsymbol{0}}$ cannot be used by any node-to-node link, including the primary links; therefore,

$$
s\left(\boldsymbol{C}_{\boldsymbol{0}} \cap \boldsymbol{C}_{\boldsymbol{i}}\right)=0, \quad \text { for } i=1,2, \ldots, k .
$$

Since the primary link carries $N$ relays' traffic, $(N . B)$ of bandwidth should be assigned to that link. Therefore,

$$
s\left(\boldsymbol{C}_{1}\right)=N \text { channels }
$$


Each $2^{\text {nd }}$ hop link is responsible for the traffic of $\left[\frac{N-1}{2}\right]$ nodes, therefore each $2^{\text {nd }}$ hop link needs $\left\lceil\frac{N-1}{2}\right\rceil$ channels. Since each node has 3 different directional antennas (one for its parent node, two for its child nodes), both antennas directed to the child nodes can use the same channel groups; therefore, for the $2^{\text {nd }}$ hop links, a total of $\left\lceil\frac{N-1}{2}\right\rceil$ channels are needed:

$$
s\left(\boldsymbol{C}_{2}\right)=\left\lceil\frac{N-1}{2}\right\rceil \text { channels. }
$$

Assuming that relays cannot transmit and receive at the same frequency at the same time, the $2^{\text {nd }}$ hop link cannot use the $N$ channels used by the primary link. Therefore,

$$
s\left(\boldsymbol{C}_{1} \cap \boldsymbol{C}_{2}\right)=0
$$

Each $3^{\text {rd }}$ hop link carries the traffic of $\left\lceil\frac{N-3}{4}\right\rceil$ relays. As for the $2^{\text {nd }}$ hop links, all of the $3^{\text {rd }}$ hop links can use the same set of channels; therefore for the $3^{\text {rd }}$ hop links, a total of $\left\lceil\frac{N-3}{4}\right\rceil$ channels are required. Since relays cannot transmit and receive on the same channel, none of the channels used in the $2^{\text {nd }}$ hop links can be assigned to $3^{\text {rd }}$ hop links. Instead, for the $3^{\text {rd }}$ hop links, since the primary links do not create interference on them and they require fewer channels than primary links, a subset of the primary link channels can be used. In the same manner, for the $4^{\text {th }}$ hop links, a subset of the $2^{\text {nd }}$ hop channels can be used. So, in general,

$$
\boldsymbol{C}_{1} \supset \boldsymbol{C}_{2 j-1} \text { and } \boldsymbol{C}_{2} \supset \boldsymbol{C}_{2 j} \quad, j=2,3, \ldots
$$

Then, from (9)

$$
\begin{aligned}
& s\left(\boldsymbol{C}_{1} \cup \boldsymbol{C}_{2 j-1}\right)=s\left(\boldsymbol{C}_{1}\right), \\
& s\left(\boldsymbol{C}_{2} \cup \boldsymbol{C}_{2 j}\right)=s\left(\boldsymbol{C}_{2}\right) \quad, j=2,3, \ldots
\end{aligned}
$$

The total number of channels can be found from (2) and (10) as follows,

$$
\begin{aligned}
s(\boldsymbol{C})= & s\left[\boldsymbol{C}_{0} \cup\left(\boldsymbol{C}_{1} \cup \ldots \cup \boldsymbol{C}_{2 j-1} \cup \ldots\right)\right. \\
& \left.\cup\left(\boldsymbol{C}_{2} \cup \ldots \cup \boldsymbol{C}_{2 j} \cup \ldots\right)\right] \quad, j=2,3, \ldots \\
= & s\left(\boldsymbol{C}_{0} \cup \boldsymbol{C}_{1} \cup \boldsymbol{C}_{2}\right) .
\end{aligned}
$$

which can further be simplified by using (5) and (8)

$$
s(\boldsymbol{C})=s\left(\boldsymbol{C}_{0}\right)+s\left(\boldsymbol{C}_{1}\right)+s\left(\boldsymbol{C}_{2}\right)
$$

Now, from equations (4), (6), and (7)

$$
s(\boldsymbol{C})=N+\left\lceil\frac{N-1}{2}\right\rceil+1 \text { channels }
$$

Finally, from (3) the relation between $W$ and $B$ can be obtained as,

$$
W=\left\{N+\left\lceil\frac{N-1}{2}\right\rceil+1\right\} \cdot B
$$

Due to the one to one correspondence between bandwidth and capacity, the relation between $R_{W}$ and $R_{B}$ can be obtained from (13) as,

$$
R_{W}=\left\{N+\left\lceil\frac{N-1}{2}\right\rceil+1\right\} \cdot R_{B}
$$

At this point we are ready to find $R_{C R N}$ from (1) and (14) as,

$$
R_{C R N} \approx\left(\frac{N}{N+\left\lceil\frac{N-1}{2}\right\rceil+1}\right)\left(M \cdot R_{W}\right) .
$$

For large values of $N$, equation (15) can be approximated by taking its limit:

$$
\begin{aligned}
& R_{C R N} \approx\left(\lim _{N \rightarrow \infty} \frac{N}{N+\left\lceil\frac{N-1}{2}\right\rceil+1}\right)\left(M \cdot R_{W}\right) \\
& \Rightarrow R_{C R N} \approx \frac{2}{3}\left(M \cdot R_{W}\right)
\end{aligned}
$$

Equation (16) is found for networks with binary trees. In general, if each root node has $p$ child nodes, then:

$$
R_{C R N} \approx \frac{p}{p+1}\left(M \cdot R_{W}\right)
$$

The equation (14) above can be interpreted as follows: cell capacity in CRN is directly proportional to the number of the root nodes; if this number is increased, then the capacity of the cell would increase and accordingly either the number of the users in the cell or the amount of the bandwidth for a user would increase. However, there is a limit on the maximum number of root nodes. To increase the capacity, all primary links use the same bandwidth at the same time. To eliminate potential interference generated by the other primary links, for each primary link, the central node has to use separate directional antenna directed to each root node, and the main lobes of those antennas should not overlap. Therefore, maximum capacity of the CRN depends on the main lobe angle of the beam antenna 
used, number of the nodes in the tree, and amount of average bandwidth used by each node.

\section{CONCLUSION}

This paper presents the capacity for Cellular Relay Networks. It is shown that the cell capacity (i.e., aggregate throughput) in CRN is almost $M$ (the number of nearest relays to the central node) times higher than that in CCN. This demonstrates the capacity increase potentials of the $\mathrm{CRN}$ can meet the high data rate coverage demand of systems beyond $3 \mathrm{G}$ in a cost effective manner. In the paper it is also found that the capacity of CRN does not depend on the size of the cell or number of hops. Since the available bandwidth is constant, the only parameter it depends on is the number of relays that directly communicate with the central node. However, if the number of relays is increased, then the area that a relay covers decreases. As a result of this, distance for the mobile user and relay communication decreases, resulting in reduced propagation losses which can be exploited by using adaptive modulation and coding.

\section{ACKNOWLEDGMENT}

This research is supported by a grant from Nortel Networks, Ottawa.

\section{REFERENCES}

[1] W. Mohr, R. Luder and K.-H. Mohrmann, "Data rate estimates, range calculations and spectrum demand for new elements of systems beyond IMT-2000", the $5^{\text {th }}$ International Symposium on Wireless Personal Multimedia Communications (WPMC), vol. 1, pp. 37-46, 2002.

[2] M. Madfors, K. Wallstedt, S. Magnusson, H. Olofsson, P.-O. Backman, and S. Engstrom, "High capacity with limited spectrum in cellular systems", IEEE Communications Magazine, vol. 35, Issue 8, pp. 38-45, 1997.

[3] H. Yanikomeroglu, "Fixed and mobile relaying technologies for cellular networks", the $2^{\text {nd }}$ Workshop on Applications and Services in Wireless Networks (ASWN), pp. 75-81, 2002. 